\title{
Postnatal Depression in Malaysia: Perspectives of Stakeholder on Awareness Medium.
}

\author{
Aishah Siddiqah A. ${ }^{\mathrm{a}, 1}$, Tuti Iryani M.D. ${ }^{\mathrm{a}}$, Mohd Abdul Wahab Fatoni M.B. ${ }^{\mathrm{b}}$, Umi Adzlin S. ${ }^{\mathrm{c}}$, Ani \\ Amelia Z. ${ }^{d}$, Marhani M. ${ }^{a}$, Hazli Z. ${ }^{a}$ \\ ${ }^{a}$ Department of Psychiatry, Faculty of Medicine, Universiti Kebangsaan Malaysia Medical Centre \\ ${ }^{b}$ Department of Syariah and Management, Academy of Islamic Studies, Universiti Malaya \\ 'Department of Psychiatry, Hospital Kuala Lumpur \\ ${ }^{\mathrm{d} D e p a r t m e n t}$ of Obstetrics and Gynecology, Faculty of Medicine, Universiti Kebangsaan Malaysia Medical Centre \\ ${ }^{1}$ Department of Psychiatry, Faculty of Medicine and Health Sciences, Universiti Putra Malaysia
}

\section{ABSTRACT}

INTRODUCTION: Postnatal depression is a highly prevailing illness, yet underdiagnosed. Untreated, it carries a transgenerational impact, and therefore promoting awareness for it has to be of high priority. This research explored the elements of an educational tool to develop awareness for postnatal depression as preferred by stakeholders. MATERIALS AND METHOD: A descriptive qualitative approach using semi-structured face-to-face interviews were conducted, where nine participants were recruited via purposive sampling from a university hospital in Malaysia. Interviews with each participant were audio-recorded and transcribed to verbatims. Emergent themes were identified during the analysis. RESULTS: Four consistent themes regarding elements for an educational tool were derived as follows: Foundations of the tool, Illness circumstances, Support bases, and Matters of perspective. CONCLUSION: An educational video within the local setting should involve the usage of a short emotionally driven video, highlighting illness- and treatment-related content, and addressing the preference and concerns of women within the religious and cultural context. The use of edutainment as an educational tool is replicable and has a high outreach even to those who are illiterate. The findings can be used in the tailored development of an educational video on postnatal depression which will benefit local women by creating awareness and encouraging early help-seeking.

KEYWORDS: postnatal depression; educational video; health awareness; qualitative study

\section{INTRODUCTION}

Postnatal depression is an underreported complication of childbirth. Mothers with this illness present with tearfulness, emotional lability, sleep and appetite disturbances, inability to cope with the infant, and

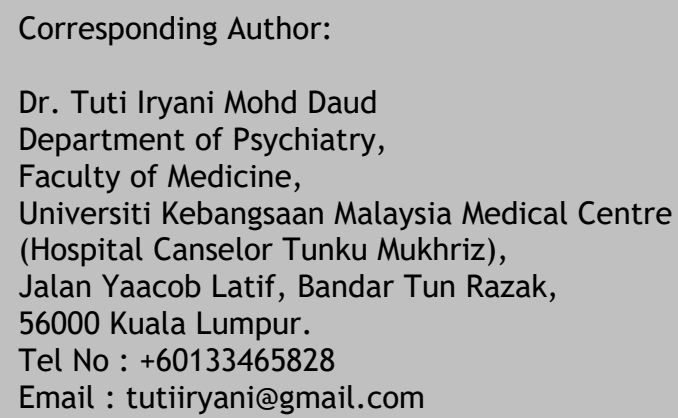

fatiguability; which remains up to two weeks or more post-delivery, and usually requires treatment. ${ }^{1}$ Some develop anxiety symptoms; worrying about the health of their newborn and blaming themselves as inadequate mothers, resulting in difficulty in bonding with her infant, and also may impact the women's interaction with her significant other and others around her.

A worldwide prevalence of $0.5-60.8 \%$ in the first 12 months of the postpartum period is seen for postnatal depression. ${ }^{2}$ Systematic reviews in low- and middleincome countries (LAMIC) show a pooled prevalence of $19.8 \% .^{3}$ In Malaysia, a prevalence of $27.3 \%$ was 
documented. ${ }^{4}$ Despite the increased prevalence, the number of women seeking help in Malaysia for postnatal depression remains unclear. International studies on help-seeking behaviour revealed that less than half of women with postnatal depression sought professional help, with women of diverse cultural backgrounds tending to reject formal services in favour of culturally sanctioned practices. ${ }^{5-7}$ Family and friends have been reported to discourage mothers from seeking formal help based on negative perceptions of past experiences with the healthcare system. ${ }^{8}$

In order to address the health beliefs of a target population, an educational tool for awareness has been found to play an important role. However, studies in LAMIC shows a lack of evidence-based educational tools that are affordable, effective, and culturally sensitive. ${ }^{9}$

This research aimed at exploring the elements of an educational tool that is suited for the Malaysian setting by speaking to stakeholders involved in postnatal depression, to gather their perspectives into the reception of education relating to postnatal depression. It aims to fill the gaps in the development of an evidence-based, locally-sensitive educational tool, tailored to the needs and wants of the target group, as an intervention to raise awareness and promote helpseeking behaviour on postnatal depression.

\section{MATERIALS AND METHODS}

\section{Approach}

A qualitative approach was employed to gain the stakeholder's perspectives on the elements of an educational tool to address issues related to postnatal depression in Malaysia. An in-depth interview was considered the best method to obtain information due to the sensitivity of the topic for an open group discussion, considering the taboo attached to speaking about personal issues in the culturally and religiously inclined Malaysian women.

A semi-structured interview guide (Table 1) was used, and participants were encouraged to be candid in their views during the face-to-face interview. As the researcher is the primary tool, interviews were conducted by ASA (corresponding author), following training by MAWFMB who is an experienced

Table 1: Sample of semi-structured questions in the interview guide.

How would women want to receive any education on postnatal depression?

How can an educational tool be made interesting for people in the community to learn from it?

What is the necessary content of such an educational tool?

What are the approaches to be used in developing such an educational tool?

\section{Research Setting}

The research took place in Universiti Kebangsaan Malaysia Medical Centre (UKMMC), Kuala Lumpur, Malaysia, with approval from UKMMC Ethics Committee. Data collection was from September 2018 until December 2018. COREQ guideline was adopted in reporting this research.

\section{Sampling and Recruitment}

Participants were recruited via purposive sampling; based on the ability to explore their knowledge and/or experience on postnatal depression.

Women with a history of Postnatal depression (diagnosis fulfilled based on DSM5) were recruited from the Psychiatry Clinic, while pregnant women were recruited from the Obstetrics and Gynaecology (ObGyn) Clinic. An advertisement about the research was made in the respective clinics to assist in recruitment. Participants were approached by treating doctors to enquire regarding their interest to participate in the research. Once agreeable, ASA sets up a time to brief the participants on the research, before interviewing them.

Meanwhile, healthcare professionals (Psychiatrist, ObGyn, and Family Medicine Specialist) were recruited via recommendations from experts of their respective fields and were approached personally in their respective workplaces. All participants were given 
information pamphlets about the research and were informed regarding the process of the interview. None of the participants approached declined or dropped out of the research.

Nine participants (Table 2) aged 23 to 45 years old, allfemale, provided written informed consent for this research. Six participants were of Malay ethnicity, two participants were Indian, and one was Chinese. Eight participants were married, while one was divorced. All the participants had completed tertiary education and were employed.

\section{Data Collection and Analysis}

Interview sessions were audio-recorded, and field notes were taken to ensure verbal and non-verbal cues were not missed. Iterative questioning was used to clarify discrepancies and gain explanations for issues mentioned by participants. ASA kept a reflexive journal of her experience within each interview to gain insight into the interaction that between her and the participant. This assisted in further discussion with co-researchers for data analysis. Triangulation of data using an interview of different participants in different settings, field observational notes, and examination of available similar research findings, was done to reduce any investigator's bias.

Recording of interviews was transcribed verbatim. Verbatims in Bahasa Melayu were translated into
English. All verbatims were verified by co-researchers. Interviews continued until data saturation. Data analysis began immediately after each interview was transcribed. During analysis, transcripts were read as a whole, allowing the data to speak on its own. This process was peer-reviewed to ensure rigor. Close discussion with co-researchers assisted ASA in gaining clarity of the available information. Initial codes help build subthemes and themes. Inconsistencies and disagreements between themes were resolved by discussion. Throughout the process of repeated reviews, themes with meaningful coherence were chosen as the grand theme. All these processes were undertaken manually by ASA, with an independent analysis done by MAWFMB to increase the trustworthiness of the research. A report was then generated (Table 3).

\section{RESULTS AND DISCUSSION}

In exploring elements required for an educational tool for postnatal depression, it is found that the type of multimedia along with the use of varying languages is pivotal in engaging the public. By using multimedia that incorporates video, graphic design, and/or audio voiceover, educational materials are easily conveyed attractively. ${ }^{10}$ Multimedia use capitalizes on providing large numbers of people with information for individualized behaviour change at a minimum cost with no training required. It even displays the potential of conveying information to those with low literacy. ${ }^{11-13}$

Table 2: Socio-demographic of participants ( $\mathrm{n}=9$ )

\begin{tabular}{|c|c|c|c|c|c|c|c|}
\hline Participant & Gender & $\begin{array}{l}\text { Age } \\
\text { (year) }\end{array}$ & Ethnicity & Population Group & Marital Status & $\begin{array}{l}\text { Education } \\
\text { Level }\end{array}$ & $\begin{array}{l}\text { Employment } \\
\text { Status }\end{array}$ \\
\hline $\mathrm{Pa}$ & F & 27 & Malay & Pregnant Woman & Married & Tertiary & Employed \\
\hline $\mathrm{Pb}$ & F & 36 & Malay & $\begin{array}{l}\text { Woman with Postnatal } \\
\text { Depression }\end{array}$ & Divorced & Tertiary & Employed \\
\hline Pc & F & 31 & Malay & Pregnant Woman & Married & Tertiary & Employed \\
\hline $\mathrm{Pd}$ & $\mathrm{F}$ & 23 & Chinese & Pregnant Woman & Married & Tertiary & Employed \\
\hline $\mathrm{Pe}$ & $\mathrm{F}$ & 41 & Indian & Healthcare Practitioner & Married & Tertiary & Employed \\
\hline Pf & $\mathrm{F}$ & 45 & Malay & Healthcare Practitioner & Married & Tertiary & Employed \\
\hline $\mathrm{Pg}$ & $\mathrm{F}$ & 38 & Indian & $\begin{array}{l}\text { Woman with Postnatal } \\
\text { Depression }\end{array}$ & Married & Tertiary & Employed \\
\hline $\mathrm{Ph}$ & $\mathrm{F}$ & 34 & Malay & $\begin{array}{l}\text { Woman with Postnatal } \\
\text { Depression }\end{array}$ & Married & Tertiary & Employed \\
\hline Pi & $\mathrm{F}$ & 42 & Malay & Healthcare Practitioner & Married & Tertiary & Employed \\
\hline
\end{tabular}


Table 3: Key elements of an educational tool for postnatal depression.

\begin{tabular}{ll}
\hline Themes & Subthemes \\
\hline $\begin{array}{l}\text { 1. Foundation of } \\
\text { Tool }\end{array}$ & $\begin{array}{l}\text { a. Approach } \\
\text { b. Content } \\
\text { c. Learning via Audio-visual }\end{array}$ \\
$\begin{array}{ll}\text { 2. Illness } & \text { a. Causal belief of postnatal depression } \\
\text { Circumstances } & \text { b. Knowledge and experience of } \\
\text { postnatal depression symptoms } \\
\text { c. Treatment of postnatal depression }\end{array}$ \\
$\begin{array}{ll}\text { 3. Support Bases } & \text { a. Role of family } \\
& \text { b. Role of husband } \\
\text { c. Role of friends } \\
\text { Perspective }\end{array}$ & $\begin{array}{l}\text { b. Cultural practice } \\
\text { c. Heligious belief }\end{array}$ \\
\end{tabular}

The format of the entertainment and content of the education is important foundations of an edutainment tool, ${ }^{14}$ a motion which is shared in this research, where the tool was unanimously preferred to be of video format.

"We can use different languages in the video and for those who can't read or write, they can visualize by using the audio and visual..." (Pg)

This research found that stakeholders preferred a single short impactful video, due to inability to maintain attention and busyness. Participants prefer to receive information quickly and effectively, and subsequently proceed with daily tasks. This concurs with findings by Wistia, a video software company, which analysed the correlation of length of video versus engagement, and found that keeping a video within two to five minutes is advisable as engagement remained steady up till then. ${ }^{15}$

"Five minutes... nothing more than that. Because you don't have enough attention or much time." (Pd)

Participants were also found to prefer an approach that would invoke a change via emotional content. Including personal narratives in an educational tool is proven to assist in symptom recognition and reducing stigma as they are an indirect form of contact with those with mental health issues. ${ }^{16}$ Studies showed that the effectiveness of an education tool depends on the presentation timing and emotional aspect of the recipient of the tool. ${ }^{17}$
"If you want to make a video, you can make it about the daily usual life of a woman, who now wonders why she feels differently after her childbirth. That is closer to the patient and they can relate to their own life. Another tactic is to avoid being strict and instead play with emotions. When something is emotional, then people will be more alert and focused." (Pa)

An effective educational tool relies on its content. This research showed that common inquiries regarding postnatal depression should be included.

"I want to know what is postnatal depression, who is suffering from it... Probably to put like a lady, you know who is suffering. What are the symptoms that she is having... and how she's being affected or what is the kind of support she has? Because people like.... most of them, like to watch negative stuff first, right? Then positive things...hmm... I want to know where can I get belp? Through whom I can get help? Is there any support group is there any number that I can contact? and it's very important for me, where can I go? Hmmm. will I be stigmatized? you know that kind of thing, I'm sure the patients want to know. And how's the support from my family if I have depression, will they love me anymore? That kind of thing... Mainly you know, women, we fear that when we have some illness whether our busband will love us or not. Correct or not? So that is very important in husband and wife relationship." (Pe)

Avoiding the use of medical jargon is another important issue raised. Healthcare practitioners often use technical medical terms in their practice, and changes are necessary to ensure that the content will be fully understood by everyone watching. ${ }^{18}$

"In my opinion, it is best not to use medical terms, because people may not understand it. Hmmm... unless we can explain the meanings of the terms. But then, some people will become disinterested once they hear or see medical terms..." (Pc)

Additionally, this research found cultural and religious elements that required highlighting in the tool. Malaysia is a diverse multifaith and multicultural country, made up of three major ethnic groups, namely Malays, Chinese, and Indians. The main religion of the country is Islam, but other faiths including Buddhism, Hinduism, Christianity among others are freely practiced. Like many Asian counterparts, Malaysians are very rooted in traditional values and beliefs. ${ }^{19}$ Strikingly, 
our research established that these beliefs and practices are tightly held even by urban educated women, especially during the postpartum confinement period.

"Confinement practices can be important for a woman to relax, eat healthy food, and get good rest. Some practices like massages help and having someone to help to cook and prepare things will ease the burden and the workload of a woman. Having the family support for confinement, or in those who can afford, getting a confinement lady belps..." (Pi)

Confinement practices may be good in instances where a woman receives support during the postnatal period. However, there is no evidence of how other practices during the confinement period may assist a woman. Our research concurred that some confinement practices could instead be a source of stress for a woman, especially when it is not tailored to the needs of the woman. ${ }^{20}$ This can be aggravated by the fact that the practices are passed down by their respected elders who these women are unable to speak up against.

"Confinement rituals are supposed to fit the individual woman. But sometimes, those who take care of the woman expect the woman to follow their ways, some of which are outdated... imagine not being allowed to wash your hair after delivery because they claim that 'wind' will enter the body. But when you think logically, if your body is beaty, you should bathe, cleanliness is more important to avoid falling ill. Also, some people are too strict with the confinement practice. For example, you have food restrictions, what kind of nutrition will you get? Imagine eating only dried anchovies every day? Anyone can experience depression. Our cultural practice can sometimes be nasty for the woman." (Pf)

Relationships of women with those surrounding her during the confinement period are paramount. ${ }^{20-21}$ This view is conveyed by participants requesting a highlight on the support system required by a postpartum woman. Some women who are less fortunate, being in marriages afflicted with affairs and domestic violence, or those struggling to maintain their relationships with overinvolved in-laws have been found to tend to develop postnatal depression. ${ }^{22}$

"Husband was the one supporting me. He persuaded me to go and see a doctor. He reminded me to think about our child - pity our son if his mom is sick. Family support is definitely important. Next would be support from friends. When we don't know anything, as first-timers, friends are the ones who are belpful, especially those with previous experiences..." (Ph)

Another interesting finding involves the need to highlight the help-seeking attitudes of Malaysian women. This research found that the urban educated women involved in this research have conservative behaviours with regards to health-decision making. We posit that this may stem from the illness itself, and possibly the patriarchy system which has been a practice in Asian countries, including Malaysia. In the patriarchal system, men hold the primary power of a family. This disparity in power has been ingrained since young and may cause a woman to be dependent on the man-figure in certain decision-making, despite being independent outside the home. ${ }^{23}$ We however agree that this presumption should not be generalized, as more women are empowered and self-efficacious.

"So, busbands don't really care, they only care for themselves. Women need to take care of themselves, women need to think. about their children. Especially our women, if you're talking about postnatal depression, it's not just postnatal depression you're talking about, it's our patriarchal culture that you need to address. Our woman is probably trained to accept more. Our culture is such that a wife should just follow what her husband says. If their husband says there is no need to go to the hospital, she will just solemnly abide. She will not go, because her husband says so... So she stays at home despite being ill. If you want to talk about postnatal depression, you need to address patriarchy. I don't know how are you going to address that though. Specifically in Malaysia, let's say our Malay women, or those who are poor, those who are financially dependent on their husband... Will they come out? Will they talk to you? Maybe, maybe not. Maybe not is much more likely, because they have no way out..." (Pb)

Additional riveting findings include the need to address misconceptions regarding mental strength and religion. Participants shared that the public has false beliefs that those experiencing postnatal depression have weak religious faith, and that praying will help them feel better. Studies have shown that religion encourages their believers to seek treatment from the experts, alongside praying and asking for healing from God. ${ }^{24}$

"It's very different here, even talking to your parents about postnatal depression, it's correlated to your religious strength, it's correlated to your status, and why are you not coping? And you 
must be some kind of crazy to see someone professional. You ask your parents, you ask your in-laws, and I don't know... even your friends would say 'Ob My God! Depression and a religious person? Probably you need to read the Quran more...' And it was in that situation that I wondered 'Am I not doing enough in terms of spirituality that I cannot overcome this..."' (Pb)

Another noteworthy content for the educational tool is the treatment concerns and preferences of women. Many women are notably concerned with taking medications due to worry about the side effects and also due to concerns about others' opinions. These women preferred to get help out of the formal hospital setting, indicating that many are still unaware of the benefits over risks of taking medications, which may help them function better. ${ }^{5}$

"Many women don't want to take medications, right? There seems to be a stigma related to psychiatry medications. Once you bring medication up, people will speak about addiction, going crasy. And then, there's the issue of breastfeeding. Some are also scared that psychiatric records may be used against them in court, especially in custody battles. That's why they don't come forward to get help. So, it is difficult..." (Pi)

Efforts to raise awareness on postnatal depression are numerous, among which include the use of pregnancy modules. However, using reading modules may be insufficient in a developing country such as Malaysia, where despite being a highly literate population (94.64\%), Malaysians have been found to have unsatisfactory reading habits. ${ }^{25}$ Efforts to increase the health literacy of Malaysians via reading may take some time, and yet issues surrounding postnatal depression are highly pressing, indicating the need for a swift and more widespread approach.

One of the ways to solve this concern would be to incorporate the use of multimedia into the pregnancy module. The use of an audio-visual tool has been proven successful in altering a person's opinion or behaviour relating to the subject matter which was screened. ${ }^{11,26}$ The advantage of using a video is in its outreach power, as people can learn directly from experts without having to travel. ${ }^{27}$

"Developing a video is very good. If we're talking about the newspaper, people don't read it nowadays. So, you can go digital.
Awareness videos can be delivered via mobile phones or TV." (Pf)

With the increasing use of the internet in current times, it is unsurprising that participants preferred the educational video to be screened on social media. Screening of video on social media advocates easy spreading of information in a dependable repeatable manner, while the stakeholders are in their familiar and comfortable setting. This helps minimise the cost of organising awareness programs and reduce the travelling cost of these groups to attend events. ${ }^{26}$

"Mostly if there are issues, then friends will keep on sharing videos on it. One person sees it, then the next one also sees it, then many friends have watched it. Then we start discussing the issue, and we will have long sharing sessions about the issue..." (Pa)

Securing on this prospect, one of the best ways forward to raise awareness and promote help-seeking behaviours with regards to postnatal depression in the Malaysian setting is the development and screening of an educational video.

\section{Strength and Limitation}

Performing qualitative research gave us an insight into a sensitive topic by looking into the opinions and experiences of the stakeholders in a more fluid and relaxed manner. However, participants involved in this research were a small group of urban employed women, and therefore, the results of this research may not be generalisable.

\section{CONCLUSION}

Despite its high prevalence with transgenerational impact, many women are unaware or reluctant to get help for postnatal depression. Developing an educational tool that incorporates elements as recommended by experts by knowledge or experience can assist in the transmission of information and reduce non-treatment. The key elements identified in this research include the use of a short, emotionally driven video, highlighting illness- and treatment-related content, avoiding medical jargon. Customising an educational video to the native needs by addressing the preferences and concerns the people have related to the 
illness and its treatment, while undertaking the religious and cultural context, using a narrative approach may result in less stigma and better reception by the public, which in return is hoped to encourage positive helpseeking behaviours.

\section{ACKNOWLEDGMENTS}

A special thanks to Miss Saleemah Alimuddin for her assistance in the study. This research was conducted in UKMMC as ASA was a masters' trainee then, while her current workplace affiliation is as mentioned above

\section{DECLARATION OF INTEREST}

None.

\section{FUNDING}

None.

\section{REFERENCES}

1. Sharma V, Sharma P. Postpartum Depression: Diagnostic and Treatment Issues. J Obstet Gynaecol Canada. 2012;34(5):436-42.

2. Norhayati MN, Nik Hazlina NH, Asrenee AR, Wan Emilin WMA. Magnitude and risk factors for postpartum symptoms: A literature review. J Affect Disord. 2015;175:34-52.

3. Fisher J, de Mello MC, Patel V, Rahman A, Tran T, Holton S, et al. Prevalence and determinants of common perinatal mental disorders in women in low-and lower-middle-income countries: A systematic review. Bull World Health Organ. 2012;90(2):139-49.

4. Mohd Arifin SR, Cheyne H, Maxwell M. Review of the prevalence of postnatal depression across cultures. AIMS Public Heal. 2018;5(3):260-95.

5. Azale T, Fekadu A, Hanlon C. Treatment gap and help-seeking for postpartum depression in a rural African setting. BMC Psychiatry. 2016 Jun 10;16(1).

6. McIntosh J. Postpartum depression: women's helpseeking behaviour and perceptions of cause. J Adv Nurs. 1993;18(2):178-84.

7. Dennis CL, Chung-Lee L. Postpartum depression help-seeking barriers and maternal treatment preferences: A qualitative systematic review. Birth. 2006;33(4):323-31.
8. Chan SWC, Levy V, Chung TKH, Lee D. A qualitative study of the experiences of a group of Hong Kong Chinese women diagnosed with postnatal depression. J Adv Nurs. 2002;39(6):5719.

9. Patel V. Mental health in low- and middle-income countries. Br Med Bull. 2007;81-96.

10. Kandula NR, Nsiah-kumi PA, Makoul G, Sager J, Zei CP, Glass S, et al. Patient Education and Counseling The relationship between health literacy and knowledge improvement after a multimedia type 2 diabetes education program. 2009;75:321-7.

11. Dahodwala M, Geransar R, Babion J, de Grood J, Sargious P. The impact of the use of video-based educational interventions on patient outcomes in hospital settings: A scoping review. Patient Educ Couns. 2018;101(12):2116-24.

12. Vandelanotte C, Mummery WK. Qualitative and quantitative research into the development and feasibility of a video-tailored physical activity intervention. Int J Behav Nutr Phys Act. 2011;111.

13. Wilson EAH, Makoul G, Bojarski EA, Cooper S, Waite KR, Rapp DN, et al. Patient Education and Counseling Comparative analysis of print and multimedia health materials : A review of the literature. Patient Educ Couns. 2012;89(1):7-14.

14. Zorica MB. Edutainment at The Higher Education as An Element for The Learning Success. In: Proceedings of EDULEARN14 Conference. 2014.

15. Fishman E. Proof That Humans Have a TwoMinute Attention Span. Wistia; 2016 [cited 2020Oct16]. Available from: https://wistia.com/ learn/marketing/optimal-video-length

16. Dwanyen L, Hans J. Postpartum Depression: Novel Use of Video-Based Interventions. J Prenat Perinat Psychol Heal. 2017;32(2):128-48.

17. Clement S, Lassman F, Barley E, Williams P, Yamaguchi S, Slade M, et al. Mass media interventions for reducing mental health-related stigma (Review). Cochrane Database Syst Rev. 2013;(7).

18. Ferguson LA. Implementing a video education program to improve health literacy. J Nurse Pract. 2012;8(8):17-22.

19. Fadzil F, Shamsuddin K, Wan Puteh SE. Traditional Postpartum Practices Among

IMJM Volume 20 No.2, Apr 2021 
Malaysian Mothers: A Review. J Altern

Complement Med. 2016;22(7):503-8. Available

from:

20. Klainin P, Arthur DG. Postpartum depression in Asian cultures: A literature review. Int J Nurs Stud. 2009;46(10):1355-73.

21. Ahmad NA, Silim UA, Rosman A, Mohamed M, Chan YY, Mohd Kasim N, et al. Postnatal depression and intimate partner violence: $\mathrm{A}$ nationwide clinic-based cross-sectional study in Malaysia. BMJ Open. 2018;8(5).

22. Jones E, Coast E. Social relationships and postpartum depression in South Asia: A systematic review. Int J Soc Psychiatry. 2012;59(7):690-700.

23. Falicov CJ. Culture, society and gender in depression. J Fam Ther. 2003;4445:371-87.

24. Koenig HG. Research on religion, spirituality, and mental health: a review. Can J psychiatry. 2009;54 (5):283-91.

25. Bernama. M'sia's literacy rate is almost $95 \%$, not 55\%: National Library: New Straits Times. NST Online. New Straits Times; 2017 [cited 2020Oct16]. Available from: https:// www.nst.com.my/news/nation/2017/05/236676/ msias-literacy-rate-almost-95-not-55-nationallibrary

26. Naslund JA, Aschbrenner KA, Araya R, Marsch LA, Unützer J, Patel V, et al. Digital technology for treating and preventing mental disorders in lowincome and middle-income countries: A narrative review of the literature. The Lancet Psychiatry. 2017;4(June).

27. Greenberg AD, Zanetis J. The Impact of Broadcast and Streaming Video in Education. 2012. Available from: http://bufvc.ac.uk/ copyright-guidance/mlr/index.php/site/403 